\title{
Morcegos do Parque Nacional do Iguaçu, Paraná (Chiroptera, Mammalia) ${ }^{1}$
}

\author{
Margareth Lumy Sekiama ${ }^{2}$ \\ Nelio Roberto dos Reis ${ }^{3}$ \\ Adriano Lúcio Peracchi ${ }^{4}$ \\ Vlamir José Rocha ${ }^{2}$
}

\begin{abstract}
Morcegos do Parque Nacional do Iguaçu, Paraná (Chiroptera, Mammalia). It's presented a survey of bat species from Parque Nacional do Iguaçu, Paraná State, it has a area of $170.086,76$ ha, located in west of the Paraná State. A total of 1403 individuals belonging to 26 species were registred.

KEY WORDS. Chiroptera, bats
\end{abstract}

Desde meados de 1930, o Estado do Paraná vem sofrendo um desmatamento intensificado. De acordo com o Instituto de Terras e Cartografia do Paraná, dos $84 \%$ de área com vegetação nativa, atualmente restaram apenas 5,2\%, constituída de "ilhas florestais", das quais as maiores áreas florestais são principalmente a Serra do Mar e o Parque Nacional do Iguaçu (LANGE \& JABLONSKI 1981).

O Parque Nacional do Iguaçu, representa o último grande remanescente de floresta pluvial subtropical do Brasil, correspondendo a mais de $1 \%$ de toda cobertura vegetal original do Estado (KOCH \& BoçON 1994), portanto, ainda pode-se encontrar um grande potencial genético neste local.

Devido à importância da área de estudo e inexistência de informações sobre a quiropterofauna local, realizou-se esse trabalho.

\section{MATERIAL E MÉTODOS}

O Parque compreende uma área de $170.086,76$ ha., situando entre $25^{\circ} 05^{\prime}$ $25^{\circ} 41^{\prime} \mathrm{S}$ e $53^{\circ} 40^{\prime}-54^{\circ} 38^{\prime} \mathrm{W}$ (IBDF 1981). Através do Rio Iguaçu no limite sul, faz fronteira com o Parque Nacional Iguazú (Argentina). Segundo a classificação de MAACK (1981), a vegetação é de dois tipos, floresta pluvial subtropical, e floresta de araucária encontrada numa pequena parte a leste do Parque. De acordo com o plano de manejo (IBDF 1981), essa unidade de conservação possui área de uso público, zona de recuperação e zona intangível (Fig. 1).

1) Contribuição número 1260 do Departamento de Zoologia, Universidade Federal do Paraná.

2) Curso de Pós-graduação em Zoologia, Departamento de Zoologia, Universidade Federal do Paraná. Caixa Postal 19020, 81531-990 Curitiba, Paraná, Brasil. Bolsista do CNPq.

3) Departamento de Biologia Animal e Vegetal, Universidade Estadual de Londrina. 86051-990 Londrina, Paraná, Brasil.

4) Departamento de Zoologia, Instituto de Biologia, Universidade Federal Rural do Rio de Janeiro. 23851-970 Seropédica, Rio de Janeiro, Brasil.

Revta bras. Zool. 18 (3): 749 - 754, 2001 


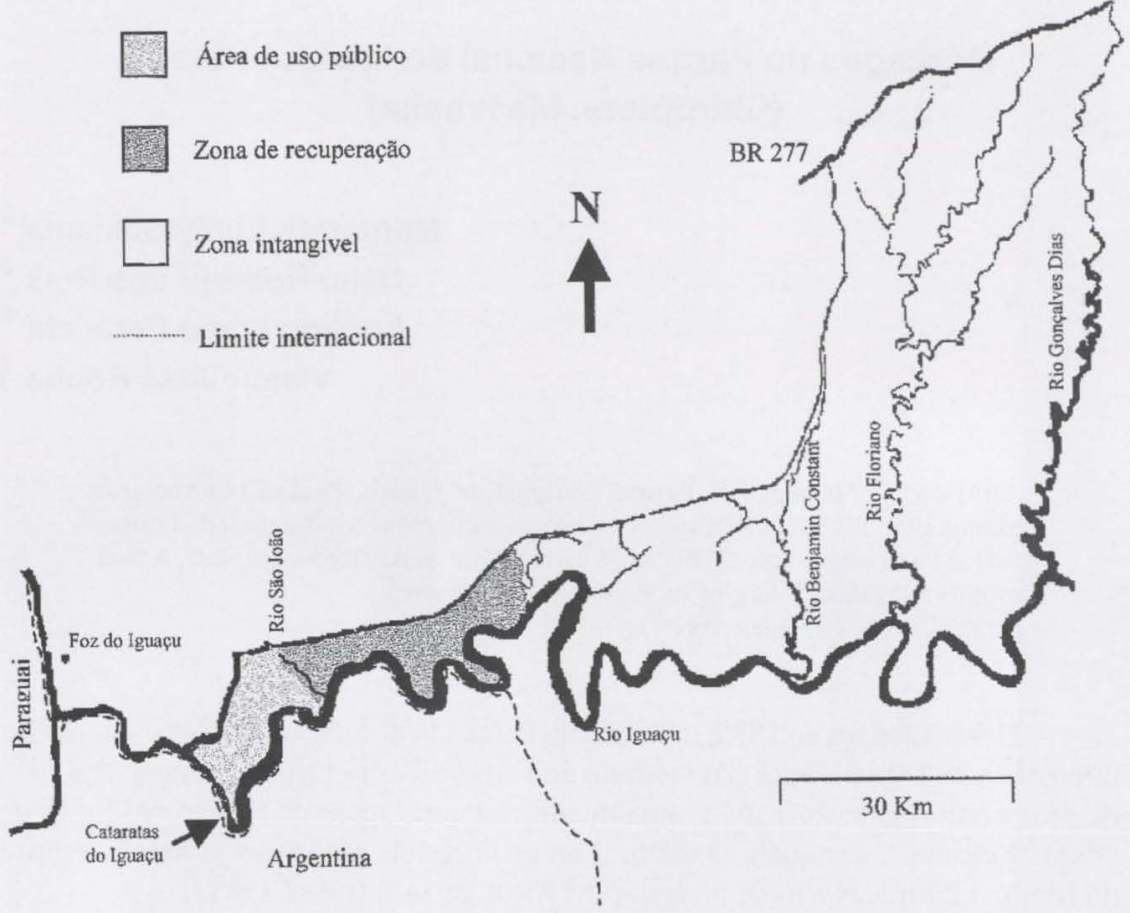

Fig. 1. Localização e zoneamento do Parque Nacional do Iguaçu, Paraná.

No período de setembro de 1998 a agosto de 2000, foram desenvolvidos trabalhos de campo utilizando redes de neblina armadas em trilhas e estradas. $\mathrm{Na}$ área de uso público e zona de recuperação (área perturbada) as coletas foram mensais, enquanto que na zona intangível (área preservada) foram sazonais. Contudo, também realizaram-se coletas manuais em casas localizadas dentro do Parque, bem como observação de morcegos em vôo. Os exemplares capturados foram medidos e pesados, e então liberados.

Exemplares de difícil identificação no campo, foram sacrificados e determinados posteriormente. Os exemplares sacrificados foram incorporados às coleções Adriano L. Peracchi, atualmente depositada no Instituto de Biologia, Universidade Federal Rural do Rio de Janeiro (ALP-UFRRJ) e no Laboratório de Zooecologia, Universidade Estadual de Londrina (LZUEL). Os exemplares expostos no Parque Nacional Iguazú (MPNI), Argentina, também foram incluídos na listagem, pois provavelmente ocorram do lado brasileiro.

\section{RESULTADOS E DISCUSSÃO}

Foram registradas 26 espécies num total de 1403 indivíduos, distribuidas em quatro famílias: 


\section{Noctilionidae}

Noctilio leporinus (Linnaeus, 1758)

Registrado através de observação direta durante comportamento de caça do morcego sobrevoando uma lagoa.

\section{Phyllostomidae \\ Phyllostominae}

\section{Chrotopterus auritus (Peters, 1856)}

Foram coletados um total de 19 machos e 12 fêmeas. Um exemplar foi conservado (LZUEL).

\section{Micronycteris sylvestris (Thomas, 1896)}

Coletado um macho sub-adulto. Primeiro registro para o Estado do Paraná. Espécie em declínio, segundo a IUCN é uma espécie presumivelmente ameaçada (NowAK 1999). Examinado um exemplar (ALP-UFRRJ).

Tonatia bidens (Spix, 1823)

Foram coletados dois machos e duas fêmeas. Conservado um exemplar (LZUEL).

\section{Glossophaginae}

\section{Glossophaga soricina (Pallas, 1766)}

Espécie registrada com base em exemplar depositado no Parque Nacional Iguazú (MPNI).

\section{Carollinae}

Carollia perspicillata (Linnaeus, 1758)

Coletados 21 machos e 15 fêmeas. Conservado um exemplar (LZUEL).

\section{Stenodermatinae}

\section{Artibeus fimbriatus Gray, 1838}

Coletados 47 machos e 41 fềmeas. Conservados dois exemplares (LZUEL).

Artibeus lituratus (Olfers, 1818)

Coletados 402 machos e 540 fêmeas. Conservados três exemplares (LZUEL).

\section{Artibeus obscurus Schinz, 1821}

Coletados dois machos e três fềmeas. Conservado um exemplar (LZUEL).

\section{Pygoderma bilabiatum (Wagner, 1843)}

Coletados nove machos e 12 fềmeas. 
Sturnira lilium (E. Geoffroy, 1810)

Coletados 108 machos e 134 fêmeas. Conservados seis exemplares (LZUEL).

\section{Desmodontinae}

\section{Desmodus rotundus (E. Geoffroy, 1810)}

Total de seis indivíduos, três machos e três fêmeas. Conservado um exemplar. (LZUEL).

\section{Diaemus youngi (Jentink, 1893)}

Espécie registrada no Parque Nacional Iguazú com base em folder distribuído pelo Parque Nacional Iguazú, Argentina.

\section{Vespertilionidae \\ Vespertilioninae}

\section{Eptesicus brasiliensis (Desmarest, 1819)}

Coletados um total de quatro machos e cinco fêmeas. Conservados dois exemplares (LZUEL).

\section{Eptesicus diminutus Osgood, 1915}

Coletado e conservado uma fềmea (LZUEL).

\section{Eptesicus furinalis (d'Orbigny, 1847)}

Espécie registrada com base em exemplar depositado no Parque Nacional Iguazú (MPNI).

\section{Lasiurus borealis (Muller, 1776)}

Coletados dois machos e uma fềmea. Conservado um indivíduo (LZUEL).

\section{Lasiurus ega (Gervais, 1856)}

Espécie registrada com base em exemplar depositado no Parque Nacional Iguazú (MPNI).

\section{Myotis levis (I. Geoffroy, 1824)}

Coletado e conservado um exemplar (ALP-UFRRJ).

\section{Myotis nigricans (Schinz, 1821)}

Coletados cinco machos e quatro fêmeas.

\section{Myotis riparius Handley, 1960}

Espécie registrada com base em exemplar depositado no Parque Nacional Iguazú (MPNI).

\section{Myotis rubra (E. Geoffroy, 1806)}

Coletado e conservado um macho (LZUEL), considerada espécie ameaçada de extinção (TADDEI \& AGUIAR 1997). 


\section{Molossidae}

\section{Molossops neglectus Williams \& Genoways, 1980}

Espécie registrada com base em exemplar depositado no Parque Nacional Iguazú (MPNI).

\section{Molossus ater E. Geoffroy, 1905}

Coletados um macho e uma fêmea em forro de casa. Conservado um exemplar (LZUEL).

\section{Promops nasutus (Spix, 1823)}

Espécie registrada com base em exemplar depositado no Parque Nacional Iguazú (MPNI).

\section{Tadarida brasiliensis (I. Geoffroy, 1824)}

Espécie registrada com base em exemplar depositado no Parque Nacional Iguazú (MPNI).

Tabela I. Número de indivíduos das espécies de morcegos em área perturbada (área de uso público e zona de recuperação) e área preservada (zona intangível) no Parque Nacional do Iguaçu.

\begin{tabular}{lccc}
\hline \multicolumn{1}{c}{ Espécies } & Área perturbada & Área preservada & Total \\
\hline Noctilio leporinus & Observação direta & - & - \\
Chrotopterus auritus & 29 & 2 & 31 \\
Mycronicteris sylvestris & - & 1 & 1 \\
Tonatia bidens & 2 & 2 & 4 \\
Carollia perspicillata & 23 & 13 & 36 \\
Artibeus fimbriatus & 73 & 15 & 88 \\
Artibeus lituratus & 782 & 163 & 945 \\
Artibeus obscurus & 5 & - & 21 \\
Pygoderma bilabiatum & 19 & 39 & 242 \\
Sturnira lilium & 203 & - & 6 \\
Desmodus rotundus & 46 & 5 & 9 \\
Eptesicus brasiliensis & 4 & - & 1 \\
Eptesicus diminutus & 1 & 2 & 3 \\
Lasiurus borealis & 1 & - & 9 \\
Myotis levis & 1 & 1 & 1 \\
Myotis nigricans & 77 & Forro de casa & - \\
Myotis rubra & - & & - \\
Molossus ater & Forro de casa & & 2 \\
\hline
\end{tabular}

A tabela I mostra que, a família Phyllostomidae foi a mais representativa, na qual $A$. lituratus e $S$. lilium foram as espécies de maior capturabilidade, seguido de A. fimbriatus. A espécie $M$. sylvestris teve apenas um exemplar capturado na zona intangível do Parque. De forma geral a família Vespertilionidae tiveram baixa capturabilidade. Enquanto $M$. ater só foi capturado manualmente em forro de casa.

A alta capturabilidade de $A$. lituratus e S. lilium no Estado do Paraná já foi relatada por MULLER \& REIS (1992); REIS \& MULLER (1995); REIS et al. (2000). Esse fato está relacionado com a metodologia, a qual de certa forma é seletiva, pois as redes são armadas ao nível das frutas disponíveis, isto é, entre 0,5 a 2,5 m do solo. Além disso, as espécies carnívoras e insetívoras, usam com mais freqüência a ecolocalização para caçar suas presas, consequentemente detectam com facilidade as redes de neblina (PEDRO \& TADDEI 1997), e também pelo motivo dos molossídeos 
voarem mais alto (HANDLEY 1967; PEDRO \& TADDEI 1997)) diminuindo a capturabilidade. Outro fato que favorece o elevado número de A. lituratus e S. lilium é a alta adaptabilidade dessas espécies à diferentes tipos de ambientes (REIS et al. 2000), como foi observado na área de uso público, onde o impacto do turismo é grande.

Quanto à Mycronicteris sylvestris, o presente trabalho relata a primeira ocorrência dessa espécie para o Paraná. Ressalta-se que, M. sylvestris e Myotis rubra foram coletadas apenas na área preservada (zona intangível) do Parque, pois além de apresentarem uma distribuição consideravelmente restrita (KOOPMAN 1982), são sensíveis à mudanças no ambiente (TADDEI \& AGUIAR 1997). Portanto, apesar da área de estudo ser uma unidade de conservação relativamente ampla (170.086,76 ha), alguns quirópteros necessitam de um ambiente com qualidade.

A Estrada do Colono localizada na zona intangível, e reaberta em 1998, é outra agressão à unidade de conservação, onde poeira, barulho e poluição dos veículos que cruzam o Parque põem em risco a vida selvagem local. Concorda-se com LYNCH \& WHIGHAM (1984) os quais relataram que a melhor estratégia de preservação das espécies, é manter a qualidade total do habitat de procriação.

AGRADECIMENTOS. Ao CNPq pelo auxilio bolsa, ao IBAMA pela autorização da realização desta pesquisa no Parque Nacional do Iguaçu e pelo apoio.

\section{REFERÊNCIAS BIBLIOGRÁFICAS}

Handley JR., C.D. 1967. Bats of canopy of na Amazonian forest. Atas Simpósio Biota Amazônica (Zoologia), Belém, Vol. 5, p. 211-215.

IBDF (Instituto brasileiro de Desenvolvimento Florestal). 1981. Plano de manejo do Parque Nacional do Iguaçu. Brasília, Fundação Brasileira para a Conservação da Natureza, 104p.

Koch, Z. \& R. Boçon. 1994. Guia ilustrado das aves comuns do Parque Nacional do Iguaçu. Curitiba, Maxi Gráfica e Editora, 38p.

Koopman, K.F. 1982. Biogeography of the bats of South America. Pymatuning Lab. Ecol. Spec. Publ., Pittsburgh, 6: 273-302.

Lange, R.B. \& E.F. Jablonski. 1981. Lista previa dos Mammalia do Estado do Paraná. Estudos Biologia, Curitiba, 6: 1-35.

LYNCH, J.F. \& D.F. WніGнлм. 1984. Effects of forest fragmentation on breeding bird communities in

Maryland, USA. Biol. Conserv., Essex, 28: 287-324.

MAACK, R. 1981. Geografia física do Estado do Paraná. Rio de Janeiro, J. Olympio, $2^{\mathrm{a}}$ ed., 450p.

Muller, M.F. \& N.R. Dos REIS. 1992. Partição de recursos alimentares entre quatro espécies de morcegos frugívoros (Chiroptera, Phyllostomidae). Revta bras. Zool. 9 (3/4): 345-355.

NowAK, R.M. 1999. Walker's mammals of the world. Baltimore, Johns Hopkins Univ. Press, $6^{\text {th }}$ ed., Vol. 1, 836p.

PEDRO, W.A. \& V.A. TADDEI. 1997. Taxonomic assemblage of bats from Panga Reserve, southeastern Brazil: abundance patterns and trophic relations in the Phyllostomidae (Chiroptera). Bol. Mus. Biol.

Mello Leitão (n. sér.), Santa Teresa, 6: 3-21.

REIS, N.R. DOS \& M.F. MULLER. 1995. Bat diversity of forests and open areas in a subtropical region of South Brazil. Ecologia Austral, Córdoba, 5: 31-36.

Reis, N.R. Dos; A.L. Peracchi; M.L. Sekiama \& I.P. DE Lima. 2000. Diversidade de morcegos (Chiroptera, Mammalia) em fragmentos florestais no Estado do Paraná, Brasil. Revta bras. Zool. 17 (3): $697-704$.

TAdDEi, V.A. \& L. Aguiar. 1997. Workshop para a conservação dos morcegos brasileiros. Chirop.

Neotrop., Belo Horizonte, 1 (2): 24-29.

Recebido em 14.IX.2000; aceito em 18.VII.2001. 